\title{
VARIATION OF LINEAR POLARIZATION IN R AQUARII SYSTEM
}

\author{
M. R. Deshpande, U.C.Joshi and A.K. Kulshrestha \\ Physical Research Laboratory \\ Navrangpura \\ A hmedabad-380009 \\ India
}

$R$ Aquarii is a very interesting symbiotic system. There is an emission nebulosity close to the star which extends in North-South direction and a "jet" feature of about 6 " with a position angle of $29^{\circ}$ has al so been observed (Sopka et al. 1982; Mauron et al. 1985; Kafatos et al. 1983). Polarization measurements are important to understand the peculiar geometry of the circumstellar material aro und the central objects. Wavelength and time dependence of pularization can be used to put constraints on the geometry of the object and to identify the mechanism(s) responsible for polarization. In view of this linear polarization measurements of $R$ Aquarii were carried out by us in UBVRI bands. Observations were made during November-December 1984 on 1 meter telescope of Indian Institute of Astrophysics, Bangalore, with a dual channel photopolarimeter discussed els ewhere (Deshpande et al. 1985). Me asured values of percent polarization and position angle at different phases alongwith the earlier observations of Serkowski (1974) and Ladjeck (1985) are plotted in Figure 1.

Figure 1 shows several interesting features -

a) In ultraviolet polarization is large and varies between 5 and 19 percent. The polarization in VRI band is small ( $\sim 0.5$ to 4 p ercent)

b) $T$ he posit ion angle in $U$ band shows small variation about the mean value $\left(\sim 120^{\circ}\right)$ with time. The position angle in VRI bands, unlike the U band, show large variation ranging $20^{\circ}$ to $170^{\circ}$.

All these observations support and may be explained through the binary model for $R$ Aquarii system in which a hot white dwarf companion orbits around a Mira variable. Detailed discussion is given els ewhere (Deshpande et al. 1986). Angle between the position angle of "jet" and mean polarization vector is $\sim 90^{\circ}$. The small variation in position angle in $U$ band around expected value of $119^{\circ}$ may be due to the precession of jet around an axis. Emission knots found at different position angle (Kafatos et al. 1983) near the star may perhaps be explained with this model. 


\section{References}

Des hpande, M. R. , Jo shi, U.C., Kulshre stha, A.K., Banshidhar,

Vadher, N. M. , Mazumdar, H.S., Pradhan, N.S. and Shah, C. R.1985. Bull. Astron. Soc. India, 13, 157.

Deshpande, M.R., Joshi, U.C., Kulshrestha, A.K. 1986. Publ. Astron. Soc. Pacific (In press).

Kafato s, M. , Hollis, J.M. and Michalitsianos, A. Ga 1983, A strophys. J. (Letters) 267, L103.

Ladbeck, R. S. 1985, A stron. Astrophys. 142, 333.

Mauron, N. Nieto, J.L., Picat, J.P. Lelivre,G. and Sol, H. 1985, A stron. Astrophys. 142, L 13 .

Serkow ski, K. 1974, I.A.U. Circul ar No. 2712.

Sopka, R.J., Herbig, G., Kafatos, M. and Michalistianos, A.G. 1982, A strophys. J. (Letter) 258, L 35.

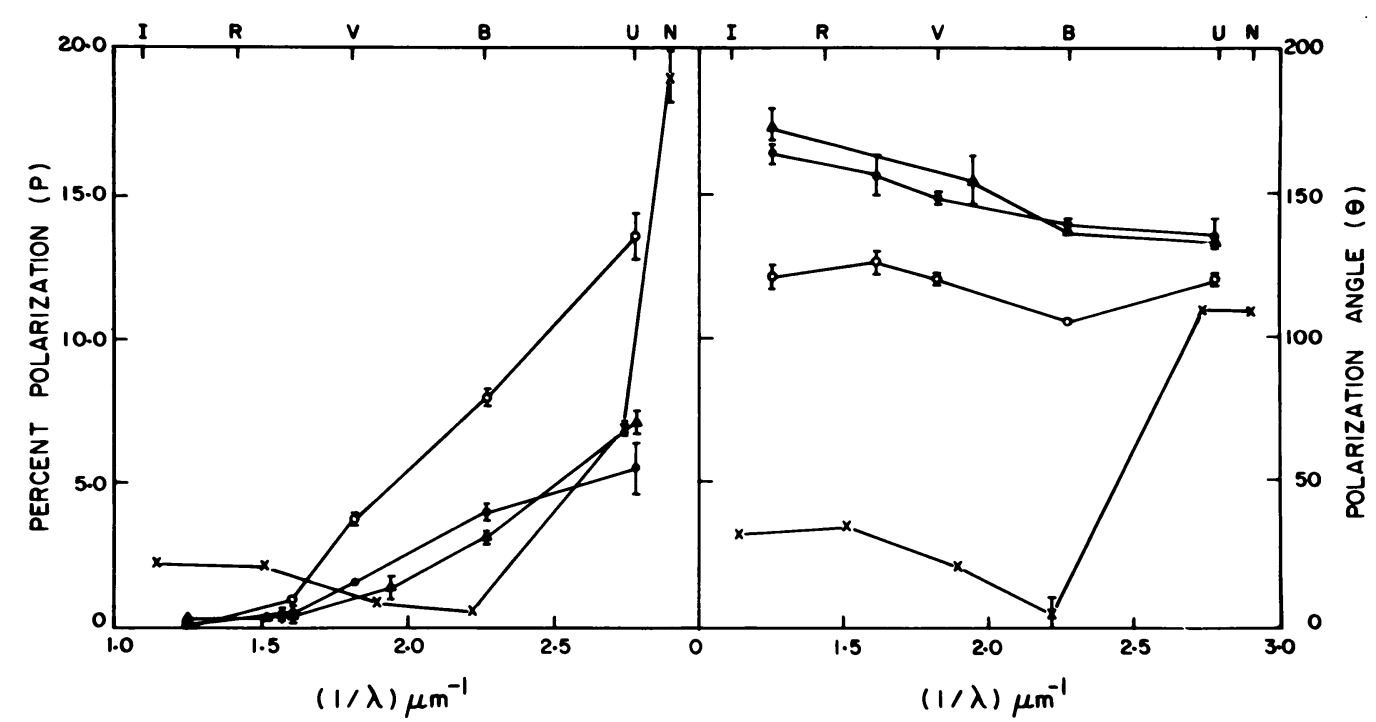

Figure 1. Plots showing wavelength dependence of polarization and position angle. Symbols represent the data taken from -

x Se rkowski (1974); L Ladbeck (1983)

- Present data (Nov. 1984); 0 Present data (Dec.1984). 